\title{
Rural Health
}

National Cancer Institute

\section{Source}

National Cancer Institute. Rural Health. NCI Thesaurus. Code C17109.

The study of health and health care delivery in rural environments. 